\title{
Patch-clamping in slices of mammalian CNS
}

\author{
Arthur Konnerth
}

A new method has been developed that permits high-resolution patch-clamp recordings in neurones from slice preparations of vitually every region of the brain or spinal cord. Since in these preparations most of the synaptic contacts are intact, the method is particularly interesting for the investigation of the molecular mechanisms of synaptic transmission at identified central neurones.

In light of the proliferation of channels and receptors that are being cloned from the mammalian CNS it is a major challenge to understand their different roles in signalling and synaptic transmission within the brain. The patch-clamp technique developed by Neher, Sakmann and co-workers ${ }^{1-3}$ has allowed successful studies of ionic currents and the underlying single-channel activity in the small cells of the CNS. The ability to voltage-clamp these cells, alter the intracellular milieu, and to record single-channel events, has clarified questions that were unapproachable with the traditional CNS electrophysiological techniques of single-unit recording and intracellular recording with conventional microelectrodes.

The patch-clamp method requires cells that have clean, smooth membranes, so it has been primarily applied to neurones in culture or to cells acutely dissociated enzymatically. Experiments on neurones within slices of brain tissue were restricted to a few special cases ${ }^{4-7}$. Recently, however, an advancement of the technique ${ }^{8}$ has allowed the method to be extended to slice preparations of almost any region of the mammalian CNS. A relatively simple 'cleaning' procedure has been developed ${ }^{8}$ that allows the exposure of neurones within thin slices ${ }^{9-11}$ of tissue. This procedure, which consists of mechanically removing the neuropile from around a neurone, circumvents the use of proteolytic enzymes that alter the properties of membrane proteins ${ }^{12}$. In addition, the experimental set-up was modified to allow for the visualization and identification of cells within slices and to provide access for recording and stimulating electrodes ${ }^{8}$.

\section{Slice preparations for patch-clamp recordings}

Slices of the brain region under study are prepared by sectioning on a vibratome. The maximum thickness of a slice is limited by the transparency of the tissue to light. Depending on the area of origin in the brain and on the age of the animal, this may vary from 70 to $150 \mu \mathrm{m}$. More recently, a standard video image intensifier, providing greater sensitivity than the human eye, has been used to allow the preparation to be viewed on a video monitor. This has extended the use of the patch-clamp to slices with more conventional thicknesses in the 300$350 \mu \mathrm{m}$ range $\mathrm{e}^{13}$

The experimental set-up used for patch-clamp recordings from slice preparations is shown in Fig. 1. A slice is placed in the recording chamber mounted on the stage of an upright microscope equipped with Nomarski differential interference contrast optics. In thin slices, single neurones can be clearly discerned by using a $\times 40$ water immersion lens. With a long working distance $(1.6 \mathrm{~mm})$ objective, it is possible to place several electrodes underneath it.

The steps involved in removing the neuropile, other neurones, glial cells and vascular tissue covering a neurone of interest are illustrated in Fig. 2. A glass micropipette with a large tip diameter (5$10 \mu \mathrm{m})$ is filled with the saline perfusing the slice and mounted in a conventional patch-pipette holder ${ }^{2}$. This 'cleaning' pipette is positioned close to the occluded neurone (Fig. 2A) while positive pressure is applied by blowing gently through the mouthpiece of the pipette holder (Fig. 2B). The resulting debris is pulled into the same cleaning pipette by applying mild suction (Fig. 2C)

The exposed plasma membrane of cleaned neuronal somas readily seals to the fire-polished tip (about $1 \mu \mathrm{m}$ in diameter) of a patch-pipette. The success rate for obtaining seals with resistances in the gigaohm range (so-called gigaseals) is comparable to that obtained with isolated cultured neurones.

\section{Identification of neurones in slices}

Normally, neurones in the upper half of a thin slice can be visualized directly (Fig. 3A). Several criteria are helpful in identifying cell types in a living tissue: (1) the relative location of the neurone within the slice; (2) the shape and size of the cell soma; and (3) the pattern of the dendritic arborization, especially the orientation of the main dendrites. These criteria were applied when identifying the hippocampal pyramidal cells from the CA1 region shown in Fig. 3A.

More detailed information about the morphology of the neurones being examined can be obtained by staining the cells with vital fluorescent dyes. Figure

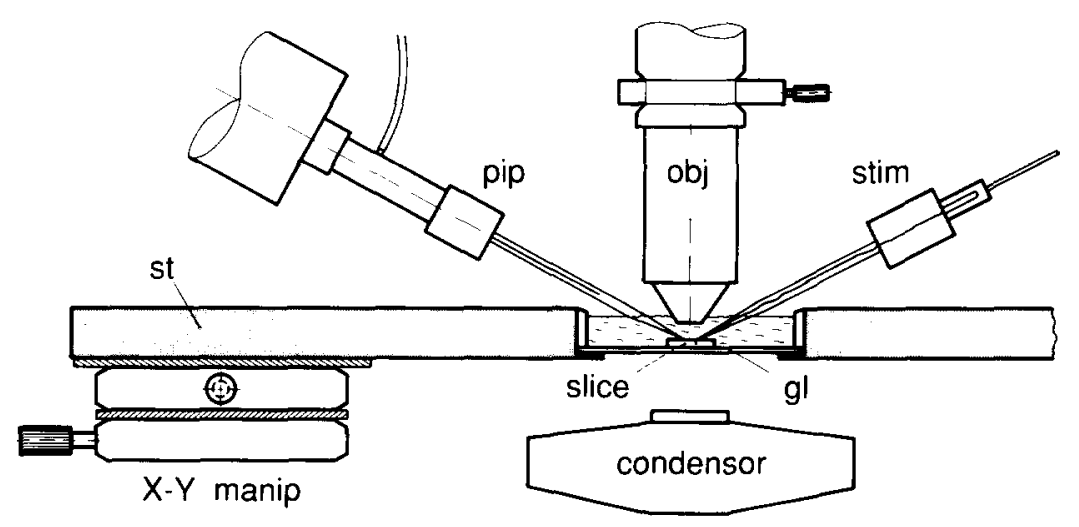

Fig. 1. Schematic diagram of the experimental set-up used for patch-clamping visually identified neurones in slices. The slice is fixed on the glass (gl) at the bottom of a recording chamber, which is mounted on the movable stage (st) of an upright microscope. The long-distance, water-immersion objective (obj) permits the simultaneous use of several micropipettes (pip, patch-or cleaning pipette; stim, stimulation pipette). Other abbreviation: $X-Y$ manip, manipulator that allows the positioning of the slice in the horizontal plane. 


\section{techniques}
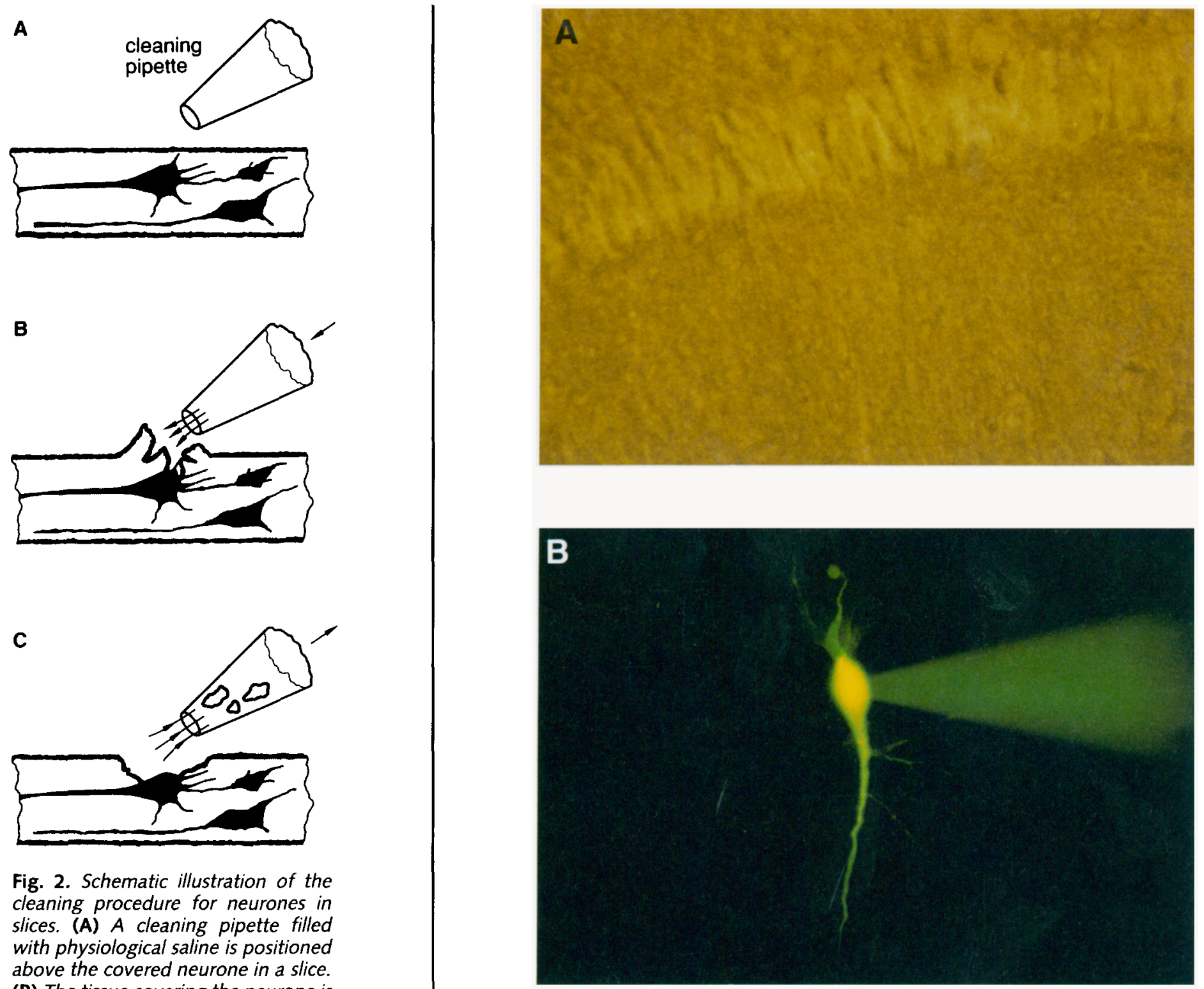

Fig. 2. Schematic illustration of the cleaning procedure for neurones in slices. (A) A cleaning pipette filled with physiological saline is positioned above the covered neurone in a slice. (B) The tissue covering the neurone is broken up by a stream of solution released by the cleaning pipette. (C) By applying gentle suction, remaining debris is pulled into the cleaning pipette. (Taken, with permission, from Ref. 8.)

Fig. 3. Photomicrographs from neurones in a 'living' rat hippocampal slice (125 $\mu \mathrm{m}$ thick). (A) Nomarski image from the surface of the CA1 region; several pyramidal cells are clearly visible. (B) Fluorescence image from the same slice after injecting a pyramidal cell with Lucifer Yellow $(0.5 \mathrm{mg} / \mathrm{ml})$. Apical and basal dendrites, as well as the dye-containing patch-pipette can be clearly seen in this plane of focus.

$3 B$ shows the result of injecting Lucifer Yellow into a CA1 hippocampal pyramidal neurone in a slice while recording its electrical activity with the patch-clamp. A second approach is to apply dyes in vivo to afferent or efferent regions of the neurones of interest a few days before slicing the brain ${ }^{8}$. The dye, transported antero- or retrogradely to specific neurones, can then be visualized in the slice to help identify specific cells.

\section{Recording synaptic currents in situ}

One of the most exciting uses of this technique involves the recording of synaptic currents within the CNS. In the tight-seal whole-cell configuration of the patch-clamp technique ${ }^{2}$, excitatory and inhibitory postsynaptic currents can be observed in cleaned neurones in brain slices. The activation of specific synaptic tracts within the mammalian CNS, and the recording of the resulting synaptic currents have so far been carried out in rat spinal cord, hippocampus and cerebellum.

In spinal cord slices, extracellular stimulation of presumed interneurones evoked inhibitory postsynaptic currents (IPSCS) in motoneurones ${ }^{8,14}$. In hippocampal granule cells, GABA-mediated IPSCS could be evoked by stimulating presumed interneurones adjacent to or within the granule cell layer of the dentate gyrus ${ }^{8,13}$. Excitatory postsynaptic currents (EPSCs) have been observed following activation of afferent fibres in the hippocampus ${ }^{15,16}$, cerebellum ${ }^{17}$ and spinal cord.

An example of EPSCs, pharmacologically isolated 


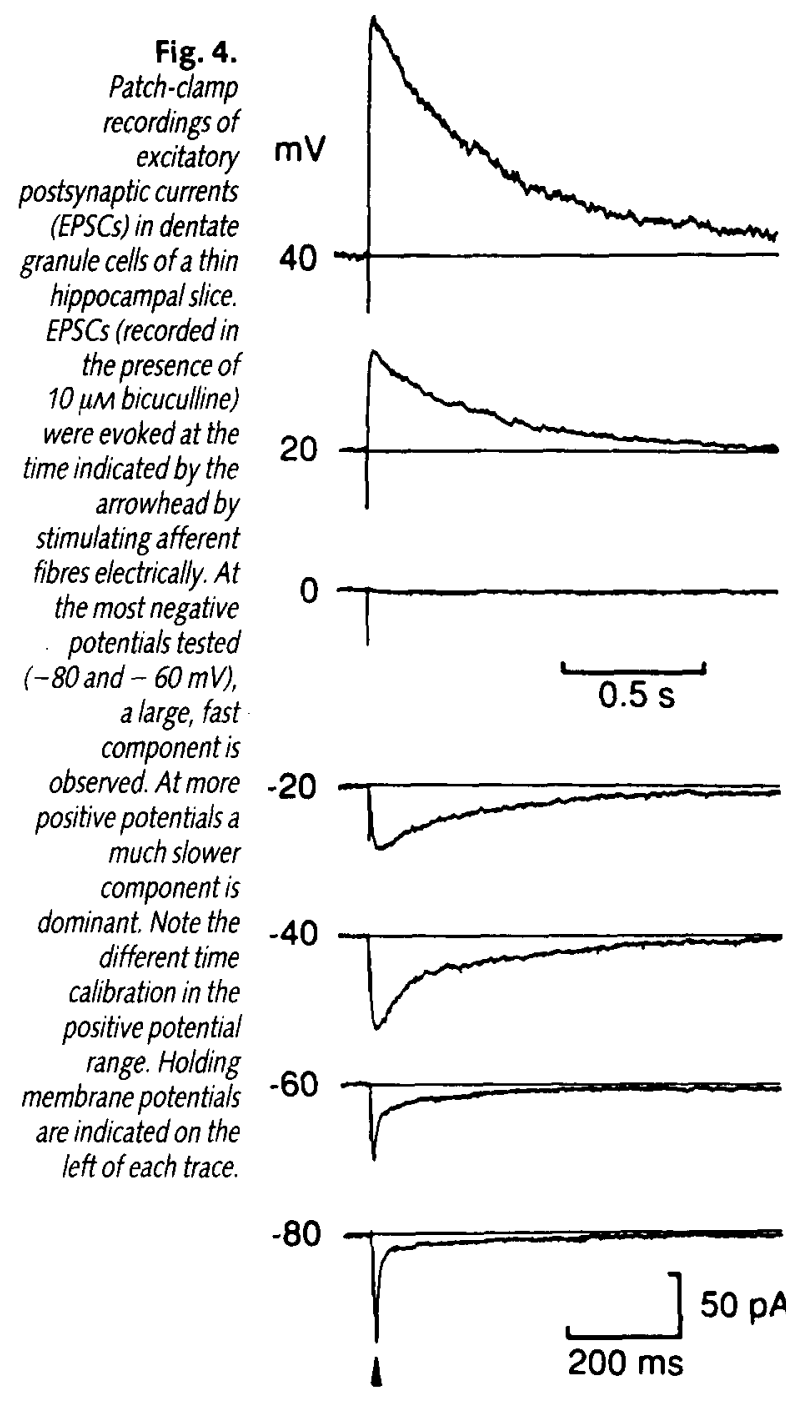

by using the GABA antagonist bicuculline, evoked in dentate granule cells of the hippocampus by afferent fibre stimulation is shown in Fig. 4. Analysis of the current traces at different holding potentials reveals a fast current component that predominates at the resting membrane potentials ( -80 to -60 $\mathrm{mV}$ ) and a slow component that predominates at more positive potentials. Pharmacological dissection of the two components indicates that they are caused by the synaptic activation of non-NMDA and NMDA receptor channels, respectively ${ }^{15}$ (Konnerth, A., unpublished observations).

\section{Conclusions and perspectives}

Application of the patch-clamp technique to neurones in slices represents a new approach for the analysis of the mammalian CNS at the molecular level. It is now possible to record whole-cell currents and the corresponding single-channel currents ${ }^{13}$ in identifiable neurones in situ from the brains of various animal species at ages ranging from newborn to adult ${ }^{8}$. This greatly facilitates the study of the mechanisms of synaptic transmission. Synaptically connected neurones can be visually identified in the living slice, and synaptic currents can be evoked by stimulating specific presynaptic neurones or fibres. This allows the functional analysis of tracts within the mammalian CNS at the single-channe level. It becomes possible, for example, to evaluate the role of the gating mechanisms of a channel protein in the control of the time course of synaptic signals, and to carry out quantal analyses of CNS synapses. The analysis of receptor-mediated responses involving intracellular messengers is enhanced by the use of the patch-pipette to control the composition of the cell. The method can be used to introduce calcium-sensitive dyes ${ }^{18}$, purified enzymes, co-factors, inhibitors, or other molecules for the investigation of second messengers involved in the short- and long-term modulation of synaptic transmission. In conjunction with scanning confocal microscopy ${ }^{13,19}$, this technique provides the opportunity to combine morphological and functional analysis of parts of neurones as small as synaptic terminals, and to map the cell membrane at the molecular level.

By allowing these types of analyses, the modification of the patch-clamp technique for recording from CNS slices provides a bridge between the morphological and electrophysiological analyses of neurotransmitter tracts and their effects on the physiology of specific cell types and the results now being generated by genetic engineering. The ability to correlate function and morphology at specific central synapses is necessary for the analysis of how the properties of specific molecules contribute to normal brain function and how changes in the expression or properties of these molecules can disrupt brain function

\section{Selected references}

1 Neher, E. and Sakmann, B. (1976) Nature 260, 799-802

2 Hamill, O. P., Marty, A., Neher, E., Sakmann, B. and Sigworth, F. J. (1981) Pflügers Arch. Ges. Physiol. 391, $85-100$

3 Sakmann, B. and Neher, E. (1983) Single-channel Recording Plenum Press

4 Gray, R. and Johnston, D. (1985) J. Neurophysiol. 54, 134-142

5 Barnes, S. and Werblin, F. (1986) Proc. Natl Acad. Sci. USA 83, 1509-1512

6 Llano, I., Marty, A., Johnson, J. W., Ascher, P. and Gähwiler, B. H. (1988) Proc. Natt Acad. Sci. USA 85, 3221-3225

7 Blanton, M. G., Turco, J. J. L. and Kriegstein, A. R. (1989) J. Neurosci. Meth. 30, 203-210

8 Edwards, F., Konnerth, A., Sakmann, B. and Takahashi, T. (1989) Pflügers Arch. Ges. Physiol. 414, 600-612

9 Yamamoto, C. (1975) Experientia 31, 309-311

10 Takahashi, T. (1978) Proc. R. Soc. London Ser. B 202, 417-421

11 Llinás, R. and Sugimori, M. (1980) J. Physiol. (London) 305, 171-195

12 McCarren, M. and Alger, B. E. (1987) Neurosci. Lett. 78, 307-310

13 Sakmann, B., Edwards, F., Konnerth, A. and Takahashi, T. (1989) Q. J. Exp. Physiol. 74, 1107-1118

14 Konnerth, A., Takahashi, T. and Sakmann, B. (1988) Eur. J. Neurosci. 1, 342 (Abstr.)

15 Konnerth, A., Keller, B., Ballanyi, K. and Yaari, Y. Exp. Brain Res. (in press)

16 Hestrin, S., Nicoll, R. A., Perkel, D. J. and Sah, P. (1990) J. Physiol. (London) 422, 203-225

17 Konnerth, A., Llano, I. and Armstrong, C. M. (1990) Proc. Natl Acad. Sci. USA 87, 2662-2665

18 Penner, R. and Neher, E. (1989) Trends Neurosci. 12, 159-163

19 Fine, A., Amos, W. B., Durbin, R. M. and McNaughton, P. A. (1988) Trends Neurosci. 11, 346-351

\section{Acknowledgements I am grateful to Drs S. DeRiemer, B. Keller and Y. Yaari for critically reading the manuscript. Work in the author's laboratory was supported by grants from the Deutsche Forschungsgemein schaft (SFB 236) and the Bundesministerium für Forschung und Technologie.}

